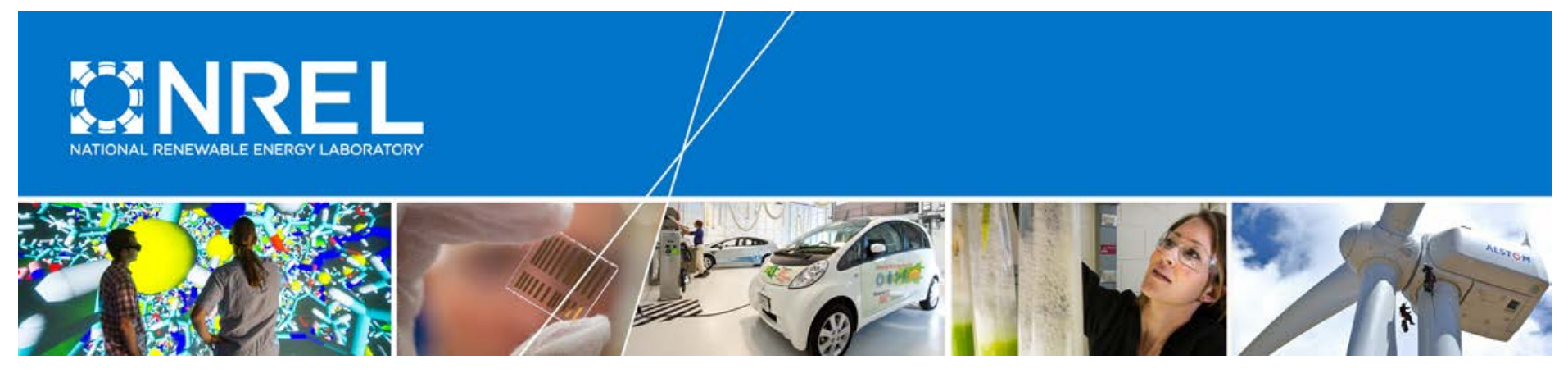

\title{
Development of a Wind Plant Large-Eddy Simulation with Measurement-Driven Atmospheric Inflow
}

\section{Preprint}

Eliot Quon and Matthew Churchfield National Renewable Energy Laboratory

Lawrence Cheung GE Global Research

Stefan Kern

GE Renewable Energy

Presented at the American Institute of Aeronautics and Astronautics SciTech 2017

Grapevine, Texas

January 9-13, 2017

NREL is a national laboratory of the U.S. Department of Energy Office of Energy Efficiency \& Renewable Energy Operated by the Alliance for Sustainable Energy, LLC

This report is available at no cost from the National Renewable Energy Laboratory (NREL) at www.nrel.gov/publications.

Conference Paper

NREL/CP-5000-67521

February 2017

Contract No. DE-AC36-08G028308 


\section{NOTICE}

The submitted manuscript has been offered by an employee of the Alliance for Sustainable Energy, LLC (Alliance), a contractor of the US Government under Contract No. DE-AC36-08GO28308. Accordingly, the US Government and Alliance retain a nonexclusive royalty-free license to publish or reproduce the published form of this contribution, or allow others to do so, for US Government purposes.

This report was prepared as an account of work sponsored by an agency of the United States government. Neither the United States government nor any agency thereof, nor any of their employees, makes any warranty, express or implied, or assumes any legal liability or responsibility for the accuracy, completeness, or usefulness of any information, apparatus, product, or process disclosed, or represents that its use would not infringe privately owned rights. Reference herein to any specific commercial product, process, or service by trade name, trademark, manufacturer, or otherwise does not necessarily constitute or imply its endorsement, recommendation, or favoring by the United States government or any agency thereof. The views and opinions of authors expressed herein do not necessarily state or reflect those of the United States government or any agency thereof.

This report is available at no cost from the National Renewable Energy Laboratory (NREL) at www.nrel.gov/publications.

Available electronically at SciTech Connect http:/www.osti.gov/scitech

Available for a processing fee to U.S. Department of Energy and its contractors, in paper, from:

U.S. Department of Energy

Office of Scientific and Technical Information

P.O. Box 62

Oak Ridge, TN 37831-0062

OSTI http://www.osti.gov

Phone: 865.576.8401

Fax: 865.576.5728

Email: reports@osti.gov

Available for sale to the public, in paper, from:

U.S. Department of Commerce

National Technical Information Service

5301 Shawnee Road

Alexandria, VA 22312

NTIS http://www.ntis.gov

Phone: 800.553 .6847 or 703.605 .6000

Fax: 703.605.6900

Email: orders@ntis.gov 


\title{
Development of a Wind Plant Large-Eddy Simulation with Measurement-Driven Atmospheric Inflow
}

\author{
Eliot W. Quon* and Matthew J. Churchfield ${ }^{\dagger}$ \\ National Renewable Energy Laboratory, Golden, Colorado, 80401, USA \\ Lawrence Cheung $\ddagger$ \\ GE Global Research, Niskayuna, New York, 12309, USA \\ Stefan Kern ${ }^{\S}$ \\ GE Renewable Energy, München, Germany
}

\begin{abstract}
This paper details the development of an aeroelastic wind plant model with large-eddy simulation (LES). The chosen LES solver is the Simulator for Wind Farm Applications (SOWFA) based on the OpenFOAM framework, coupled to NREL's comprehensive aeroelastic analysis tool, FAST. An atmospheric boundary layer (ABL) precursor simulation was constructed based on assessments of meteorological tower and radar data over a 3-hour window. This precursor was tuned to the specific atmospheric conditions that occurred both prior to and during the measurement campaign, enabling capture of a night-to-day transition in the turbulent ABL. In the absence of height-varying temperature measurements, spatially averaged radar data were sufficient to characterize the atmospheric stability of the wind plant in terms of the shear profile, and near-ground temperature sensors provided a reasonable estimate of the ground heating rate describing the morning transition. A full aeroelastic simulation was then performed for a subset of turbines within the wind plant, driven by the precursor. Analysis of two turbines within the array, one directly waked by the other, demonstrated good agreement with measured time-averaged loads.
\end{abstract}

\section{Introduction}

H IGH-fidelity flow simulations have become an essential part of the wind plant engineering process, from H preliminary site assessment to performance analysis of operational wind plants. ${ }^{1,2,3,4}$ Given the abundance of available data, it is possible to improve on our computational models by incorporating actual time-varying field data. The inherent challenge lies in synthesizing the data in a meaningful and consistent manner. For instance, separating turbulence fluctuations from the mean signal can be a difficult task given differences in measurement type and sampling resolution (spatial and temporal) between available data sets. Moreover, the atmospheric boundary layer (ABL) in which the wind plant operates changes stability over the course of a day, resulting in different wind turbine dynamics. ${ }^{5}$ Depending on the simulation length and time of day, accurate capture of atmospheric physics must account for the diurnal cycle. Assimilating field measurements and perturbing the mean flow without causing the simulated ABL state to deviate from observations is therefore critical.

Calculating a flow field in the ABL with large-eddy simulation (LES) requires a turbulent inflow that may either be generated from an engineering model or simulated by a precursor simulation. The second approach resolves the turbulence on a laterally periodic domain assuming a fully developed boundary layer, ${ }^{6}$ and is the approach employed here. Typically, a number of parameters that specify initial and boundary conditions are tuned to achieve an ABL with a desired turbulence intensity (TI). These simulations generally

\footnotetext{
*Postdoctoral Researcher, National Wind Technology Center, 15013 Denver West Parkway, AIAA Member.

$\dagger$ Research Engineer, National Wind Technology Center, 15013 Denver West Parkway, AIAA Member.

$\ddagger$ Mechanical Engineer, 1 Research Circle, AIAA Member.

$\S$ Senior Engineer, Freisinger Landstraße 50, AIAA Member.
} 
Table 1. Summary of data available during the period of investigation.

\begin{tabular}{lcccc}
\hline Data Set & Wind Data & $\begin{array}{c}\text { Temperature } \\
\text { Data }\end{array}$ & $\begin{array}{c}\text { Spatial } \\
\text { Resolution }\end{array}$ & $\begin{array}{c}\text { Temporal } \\
\text { Resolution }\end{array}$ \\
\hline Radar (4 km x 4 km) & $\checkmark$ & & $10 \mathrm{~m}$ & $1 \mathrm{~min}$ \\
Met Mast & $\checkmark$ & $\checkmark$ & $19.25 \mathrm{~m}$ & $1 \mathrm{~s}$ \\
Lidar (3 units) & & $\checkmark$ & $\mathrm{n} / \mathrm{a}$ & $2 \mathrm{~min}$ \\
\hline
\end{tabular}

assume a constant hub-height wind speed and direction. A problem arises when the atmospheric stability needs to be specified in addition to the turbulence level; in this case, the LES may reach an equilibrium state that does not correspond to the desired conditions. For instance, in a modeling scenario with high shear and high TI, the LES may converge to a solution with low TI as a result of surface cooling, thereby describing an atmosphere that is too stable.

In this paper, we attempt to characterize the flow field of a wind plant located in the Midwest region of the United States. The site is located on flat terrain with a variable crop height and is instrumented with a meteorological tower (met mast), radar, and three lidar units. This study focuses on a set of measurements collected between 8:15 a.m. and 11:26 a.m. local time, during which scattered, broken, and overcast conditions were observed at a nearby weather station. Since this time period occurred shortly after sunrise (at 7 a.m.) while the local atmosphere was heating up, the transition from nighttime conditions should be modeled. The objectives for utilizing these data, outlined in Sections II-IV, respectively, are to:

1. Characterize changes in wind speed, wind direction, temperature, and atmospheric stability during the measurement campaign;

2. Develop a turbulent precursor simulation with the same time-varying atmospheric characteristics prior to and during the measurement period; and

3. Simulate operational turbines subject to this turbulent inflow.

The LES simulations in this work were performed using the Simulator for Wind Farm Applications (SOWFA) tool set ${ }^{7}$ developed at the National Renewable Energy Laboratory (NREL). This simulator is based on the OpenFOAM framework and is coupled to NREL's FAST ${ }^{8}$ tool for comprehensive aeroelastic analysis including turbine structural dynamics and control. The general simulation workflow involves two main parts. First, a precursor is performed to fully develop turbulence within the LES, based on the atmospheric conditions identified in Section II. This simulation does not include any modeled turbines and is run until quasi-equilibrium is reached. To model a night-to-day (or approximately stable-to-unstable) transition, the precursor simulation is then continued with a constant heating rate applied at the ground. The resultant precursor flow field is sampled and mapped to the inlet boundary of the main simulation. Second, a main simulation including actuator-line models of the wind turbines is performed. For this calculation, the interior of the mesh is refined to resolve the turbines and their wakes. This paper focuses on development of the precursor simulation to match measured atmospheric conditions and presents sample results from a turbine-turbine interaction simulation subject to these conditions.

\section{Flow Field Characterization}

A number of atmospheric quantities need to be estimated to define the SOWFA simulation parameters, including the time-varying wind vector, turbulence level, and atmospheric stability. In the present work, these estimates are driven by available measurements during the period of interest, summarized in Table 1 by type and associated spatio-temporal resolution. Dual-Doppler radar data were acquired over a 4-kmby-4-km area, illustrated in Fig. 1. This flow field was mapped to a region 40-120 m above the ground to account for terrain effects. Instrument sensitivity to changing weather conditions resulted in a 25-min gap in data starting at 8:51 a.m., approximately 30 minutes into the measurement campaign. High-frequency met mast data were also obtained, including wind speed from cup anemometers at three different heights, wind direction at hub height, and temperature at an unknown height. Velocity measurements were quantized in approximately $2 \mathrm{ft} / \mathrm{s}$ bins. Lastly, temperature data with $0.1^{\circ} \mathrm{C}$ precision were available from lidar-mounted sensors. Lidar wind data were not available during this time. 


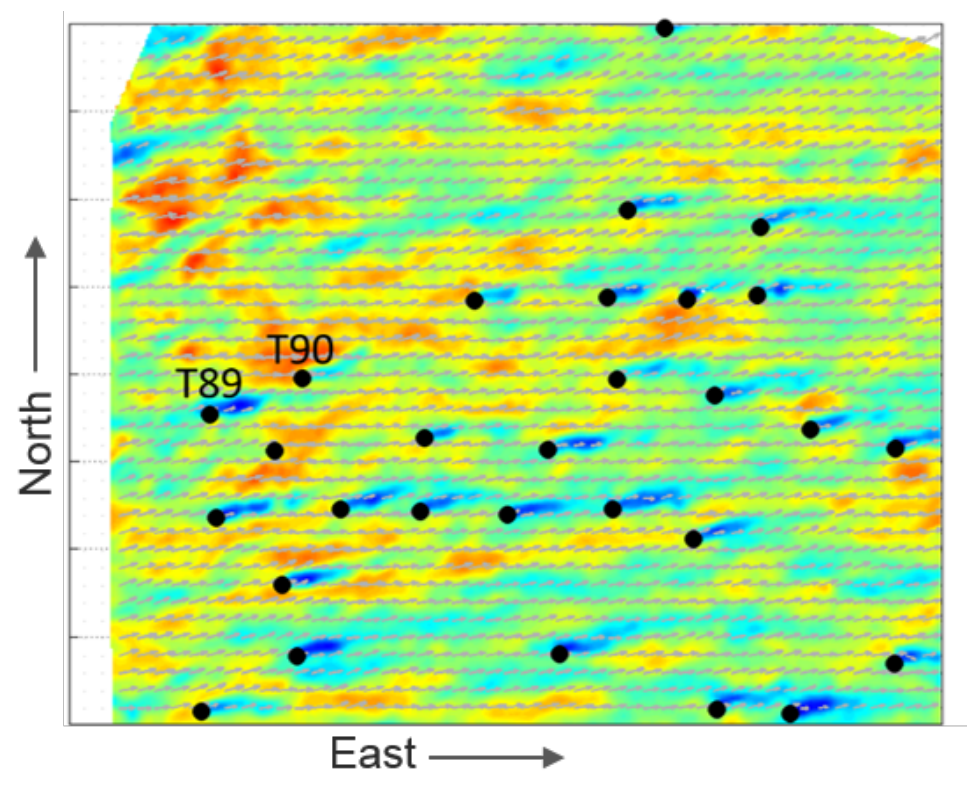

Figure 1. Wind farm layout showing the locations of turbines T89 and T90.

\section{II.A. Mean Wind Speed}

Wind speed measurements are available from two sources: the met mast and radar. To produce a continuous radar velocity field, an inpainting algorithm was applied to iteratively replace not-a-number (NaN) values in the data with a Gaussian-weighted average of surrounding non-NaN values. To estimate the height-varying velocity at the same locations as on the met mast, upwind radar data from the western edge of the measured region (Fig. 1) were sampled and spatially averaged along horizontal lines. Figure 2 compares the lineaveraged radar data (plotted in black) with instantaneous velocities measured at three locations on the met mast corresponding to the hub height, midblade height (below the hub), and blade-tip height (below the hub). Ten-minute averages of the met mast data were also calculated and are overlaid in an inverse color in Fig. 2. Good agreement between averaged measurements was observed, with the root-mean-squared (RMS) difference between measurements at the low, middle, and high positions being $0.41,0.36$, and $0.32 \mathrm{~m} / \mathrm{s}$, respectively. While both measurements are viable for specifying the simulation inlet velocity, the radar data have greater spatial resolution and are assumed to be more representative of the inflow region as a whole. The time-varying, precursor-driven inlet boundaries for the main simulation (described in Section IV) are therefore specified from the averaged radar measurements.

\section{II.B. Turbulence Intensity}

Estimates of the turbulence level require high temporal resolution and therefore were only calculated using met mast data (sampled at approximately $1 \mathrm{~Hz}$ ). Comparisons between the radar and met mast have indicated that the met mast point measurements of wind speed are representative of the flow over the greater inflow region, with an RMS difference of at most $0.41 \mathrm{~m} / \mathrm{s}$. Therefore, we expect the met-mast TI to be a reasonable representation of the turbulence in the region. TI is calculated from the standard deviation in measured velocity over 10-minute windows of instantaneous data (plotted in Fig. 2). The results in Fig. 3 indicate that the simulated TI should range from about $15 \%$ close to the ground $(z \approx 40 \mathrm{~m})$ to $12 \%$ at hub height.

\section{II.C. Mean Temperature}

The temperature measured on the met mast gradually increases through the analysis time period (Fig. 4) and is expected to be associated with the transition from nighttime to daytime (approximately stable to unstable) conditions in the ABL. During the first part of the radar measurement campaign, the temperature reported by the met mast was approximately constant at $8.2^{\circ} \mathrm{C}$; in the second part, the temperature appeared 

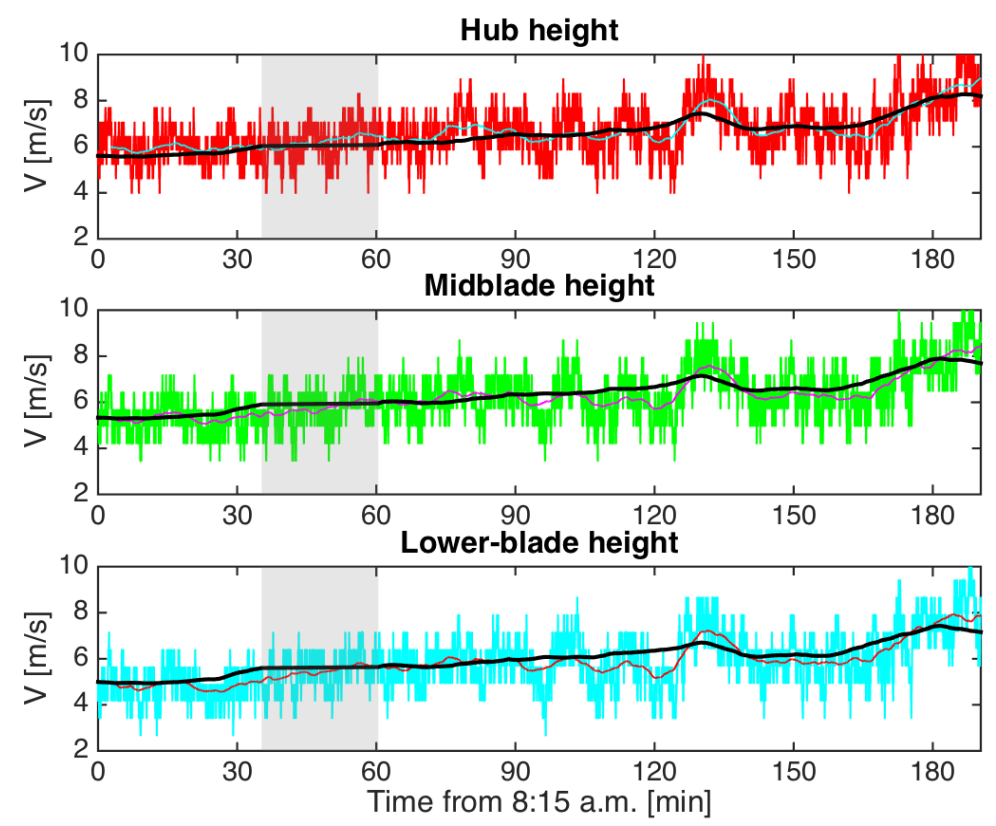

Figure 2. Velocity measurements from the radar (black) and met mast (red, green, and blue).
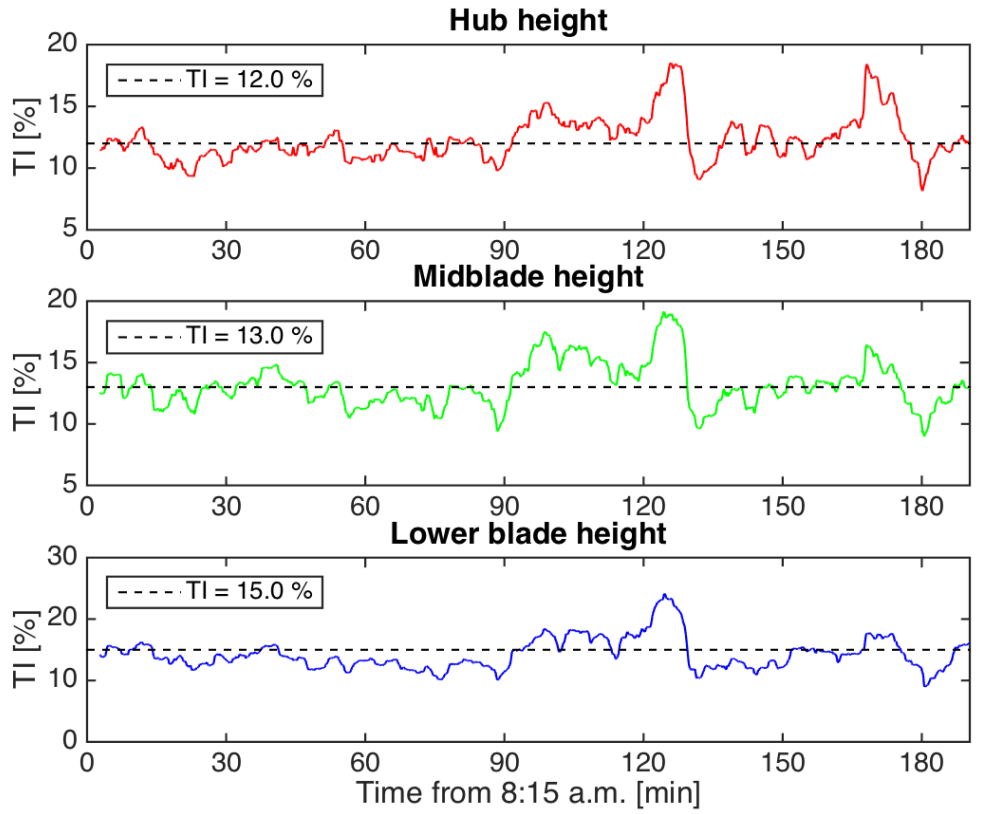

Figure 3. Estimated turbulence intensity from met mast measurements. 


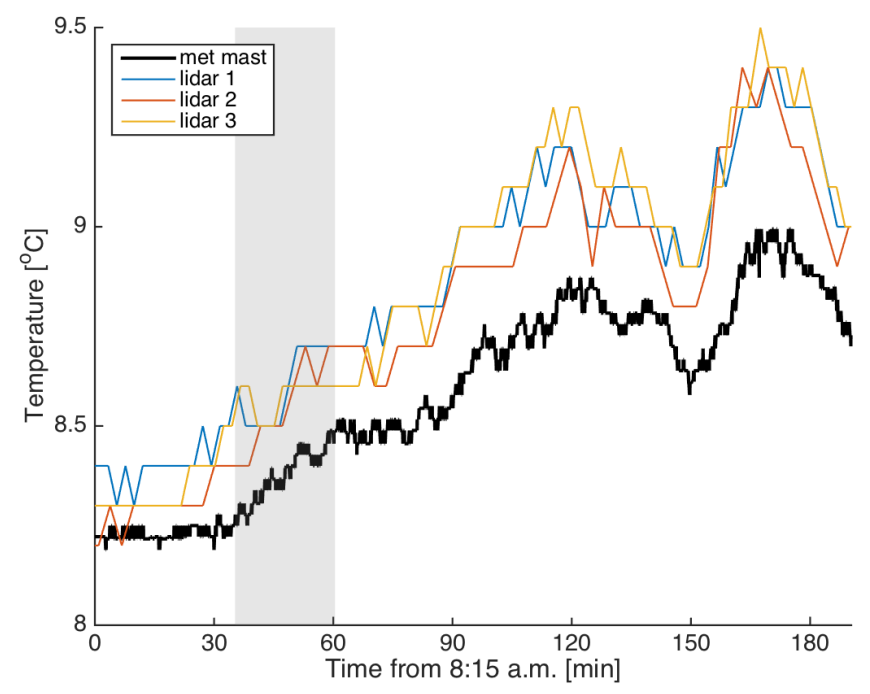

Figure 4. Temperature measurements from the met mast and lidar units. The period during which no radar data were recorded is shaded in grey.

to increase linearly with time up to $8.8^{\circ} \mathrm{C}$. The exact height at which these data were collected on the met tower is not known. In contrast, the lidar temperature data come from an external met antenna located 1.5 $\mathrm{m}$ above ground. These measurements do not clearly show a change in weather but demonstrate a similar warming trend with an approximate $+0.2^{\circ} \mathrm{C}$ bias, likely due to a difference in measurement height. Because the temperature sensors on the lidars are near to the ground, the average of the three sensors has been used to estimate the constant ground heating rate to be simulated during the measurement period.

\section{II.D. Wind Shear}

As there is neither sufficient temperature data to estimate the temperature gradient near the ground nor measurements of heat flux, we were unable to quantify the atmospheric stability in terms of Richardson number. Instead, we have inferred changes in stability from changes in wind shear. Using 10-minute averages of the wind speed measured at three heights (corresponding to the locations of the met mast probes), we calculated an instantaneous power law wind profile:

$$
u(z)=u_{\text {ref }}\left(\frac{z}{z_{\text {ref }}}\right)^{\alpha}
$$

The reference velocity and height $\left(U_{\text {ref }}\right.$ and $\left.z_{r e f}\right)$ are specified from the time-averaged hub-height velocity and the resulting shear-exponent history is plotted in Fig. 5. Two notable time periods corresponding to the changing weather conditions can be clearly identified from the met-mast-estimated shear, with the radar blackout occurring in the shaded region of the figure.

For comparison, the shear exponent has also been calculated using the radar data. Because the objective of this exercise is to generate turbulent inflow data, radar data were sampled at locations coincident with the inlet of the simulation domain. The data on this plane have been averaged in the horizontal direction at the same locations as the met mast to provide a spatially averaged, time-varying velocity profile. These profiles were also fit to Eq. (1) to provide the radar-estimated shear. The change in weather conditions is less clear from these data.

In general, the shear exponent $(\alpha)$ ranges between 0.1 and 0.2 for a nearly neutral atmosphere, ${ }^{9}$ where larger and smaller values are indicative of stable and unstable conditions, respectively. The met mast data show a pronounced transition from a stable to a nearly neutral atmosphere, while the radar data indicate near-neutral conditions throughout. It is not immediately apparent which set of initial conditions at 8:15 a.m. is most appropriate in the current study. This issue will be further discussed in Section III. 


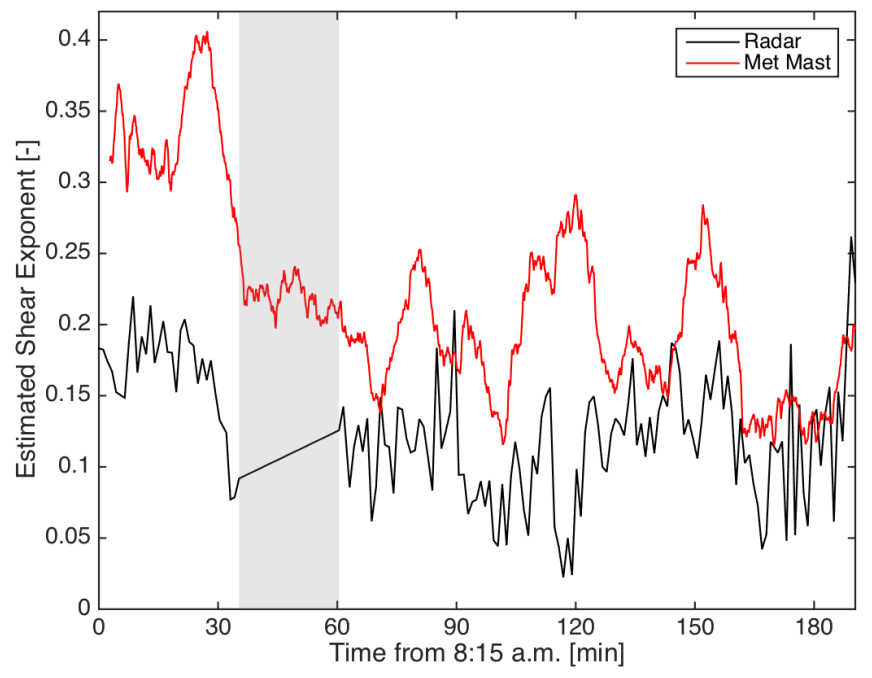

Figure 5. Estimated shear exponent from field measurements. The period during which no radar data were recorded is shaded in grey.

Table 2. Summary of SOWFA parameters affecting the atmospheric state.

\begin{tabular}{lccc}
\hline Parameter & Expected Range & Initial Guess & Final Value \\
\hline Ground conditions & & & \\
Surface roughness, $z_{0}$ & $0.03-0.25$ & $0.1 \mathrm{~m}$ & $0.2 \mathrm{~m}$ \\
Initial heating rate & $0-\mathcal{O}(1)^{\circ} \mathrm{K} / \mathrm{hr}$ & $-1^{\circ} \mathrm{K} / \mathrm{hr}$ & $0^{\circ} \mathrm{K} / \mathrm{hr}$ \\
Assumed initial atmospheric stability & $\mathrm{n} / \mathrm{a}$ & stable & neutral \\
\hline Inlet conditions & & & \\
Inversion height & $\mathcal{O}\left(10^{2}\right)-\mathcal{O}\left(10^{3}\right)$ & $500 \mathrm{~m}$ & - \\
Upper temperature gradient & $\mathcal{O}(0.1)-\mathcal{O}(1)^{\circ} \mathrm{K} / \mathrm{km}$ & $1^{\circ} \mathrm{K} / \mathrm{km}$ & - \\
\hline
\end{tabular}

\section{Precursor Development}

The computational domain size was set to $5 \mathrm{~km}$ by $3 \mathrm{~km}$ in the horizontal extents and $1 \mathrm{~km}$ in the vertical extent. A uniform cell size of $10 \mathrm{~m}$ was chosen based on prior work ${ }^{5}$ with second-order central differencing applied. For time integration, we used a second-order backward scheme with an adjustable time-step size to maintain a maximum Courant number below 0.75. In practice the actual time-step size ranged between 0.4 and $0.7 \mathrm{~s}$ per step.

Table 2 summarizes the SOWFA precursor inputs for tuning to the turbulence level and atmospheric stability identified in Section II. The ground conditions were applied to calculate the surface temperature flux whereas the inlet conditions were only used to initialize the precursor field. A weak temperature inversion was assumed to occur at a low height $(0.5 \mathrm{~km})$ indicative of nighttime or early-morning (before sunrise) conditions. At the ground, the relevant parameters include surface roughness and heating rate: surface roughness is terrain-dependent, ranging from 0.03 for a fallow field ${ }^{10}$ up to 0.25 for mature crops, ${ }^{11}$ whereas heating rate is highly dependent on the current atmospheric state. The assumption of stable or neutral stability in the early morning dictates the constants in the Monin-Obukhov velocity and temperature scaling laws. Under neutral conditions, the friction velocity is directly calculated from the log law, whereas stable or unstable conditions require an iterative solution approach. ${ }^{12}$

\section{III.A. Precursor Phase 1}

The first phase of the precursor simulates the period of time in the early morning leading up to the start of the measurement period. We assume that the wind speed, wind direction, and ground heating rate were 
Hub height

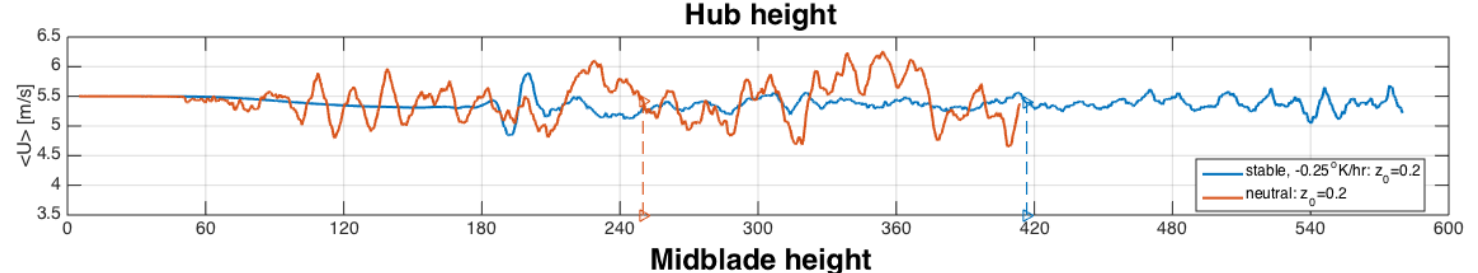

Midblade height
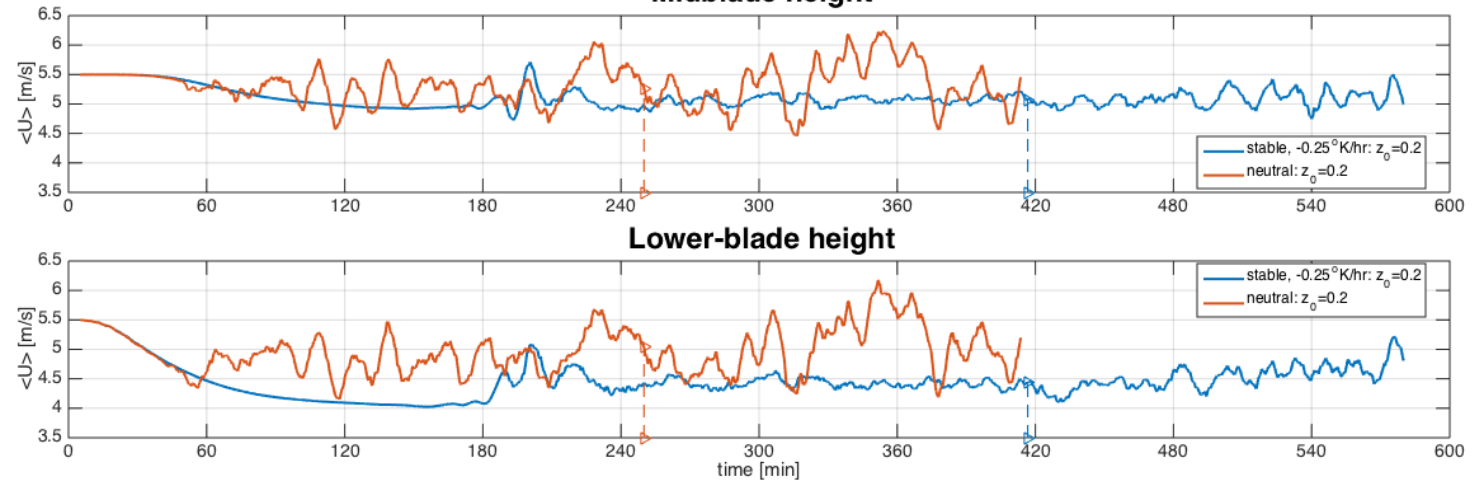

(a) Mean Velocity
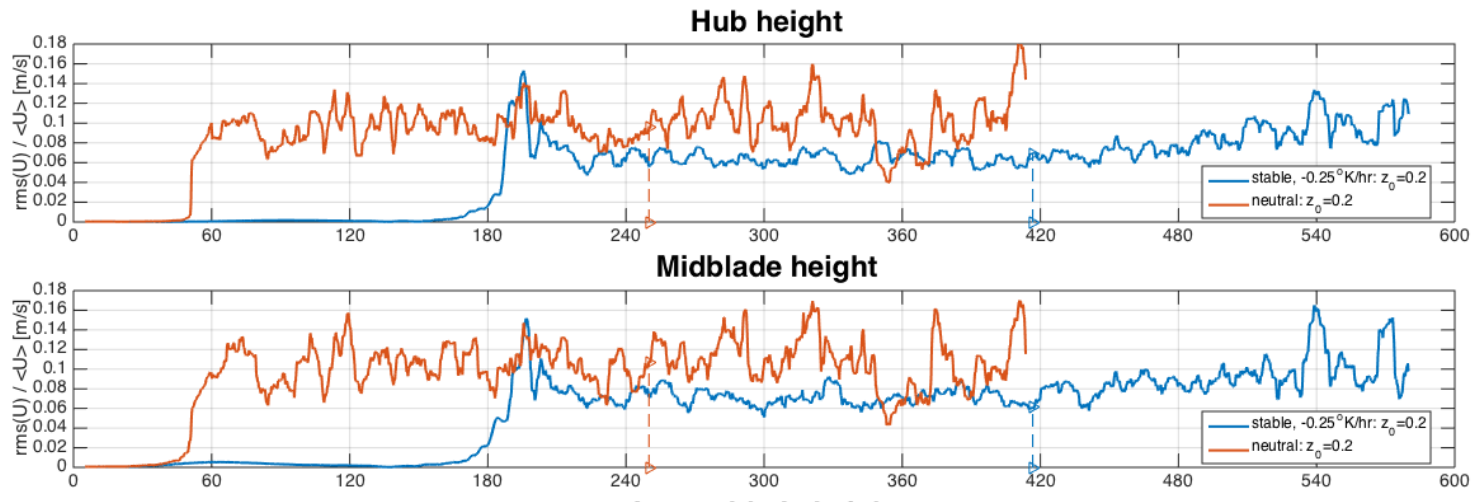

Lower-blade height

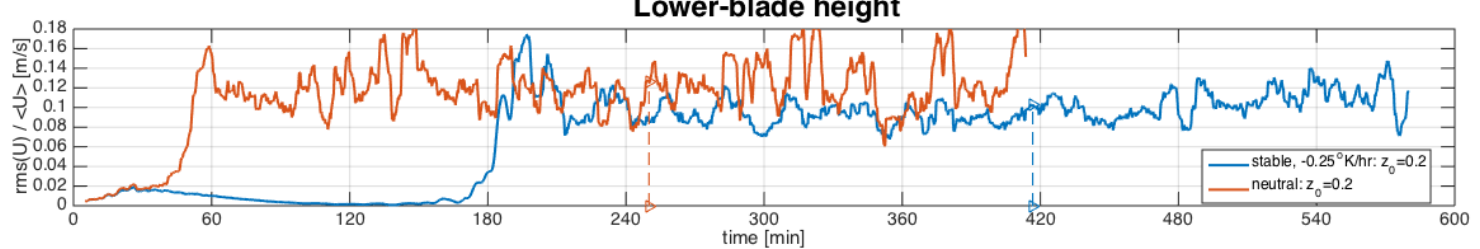

(b) Turbulence Intensity

Figure 6. Simulated results from precursor phases 1 and 2 for different initial stability states. Constant heating (during phase 2) was applied after the times indicated by the vertical dashed line.

This report is available at no cost from the National Renewable Energy Laboratory (NREL) at www.nrel.gov/publications. 
constant during this time. However, the atmospheric stability state at the start of the measurement period is unknown, as evidenced by the different shear estimates in Fig. 5. We therefore simulated both stable and neutral starting conditions with the initial guesses noted in Table 2. The surface roughness height was estimated to be $0.1 \mathrm{~m}$ based on reported ground conditions at the wind plant. However, the crop fields in the vicinity of the turbines were known to be non-uniform and no record exists detailing which fields in the area had been harvested at the time. An initial estimate of $1^{\circ} \mathrm{K} / \mathrm{hr}$ was used for the surface cooling rate based on previous experience with stable atmospheric conditions. Stable calculations were performed for 25,000 seconds whereas the neutral calculations were only performed for 15,000 seconds (417 and 250 minutes, respectively). Results are plotted in Fig. 6, with the end of precursor phase 1 (demarcated by vertical dashed lines) corresponding to the beginning of the measurement period.

We first observed that the initial cooling rate in the stable case was causing the simulated ABL to laminarize, resulting in a turbulence level of approximately zero. To promote the development of turbulence in this case, the cooling rate was reduced by a factor of four to $0.25^{\circ} \mathrm{K} / \mathrm{hr}$. A turbulent $\mathrm{ABL}$ was formed, characterized by a wind shear profile with $\alpha \approx 0.3$. This value is comparable to the shear exponent estimated from the met mast (Fig. 5). The difference in shear between the stable and neutral cases is observed in the reduced velocity near to the ground in the stable case (Fig. 6a). However, the simulated turbulence intensity was significantly lower than estimated (Fig. 3): the near-ground TI was about $10 \%$ and hub-height TI was only about $6 \%$, half the target value (Fig. $6 \mathrm{~b}$ ). The roughness was subsequently increased from 0.1 to 0.2 $\mathrm{m}$, and then to $0.5 \mathrm{~m}$, to increase TI. However, the effect on the stable precursor results was negligible, indicating that turbulence was effectively suppressed by heat removal through the ground. In the neutral case, increasing the roughness height from 0.1 to $0.2 \mathrm{~m}$ increased the hub-height TI to approximately $10 \%$; a further increase in roughness from 0.2 to $0.5 \mathrm{~m}$ increased the TI by a negligible amount. A final roughness height of $0.2 \mathrm{~m}$, lying within the expected range for crops, was therefore selected.

To achieve the best possible agreement between measured and simulated turbulence intensity under the measured conditions, we focus on simulating a neutral atmosphere and set the phase 1 heating rate to zero. The resulting average shear exponent was approximately 0.15 , comparable to the radar measurements in Fig. 5. There appears to be no straightforward approach for matching the simulated turbulence level from the bottom edge of the rotor up to hub height for neutral or slightly stable conditions. With the hub-height TI differing from met-mast measurements by at most $2 \%$ in the neutral case, we proceed to the next phase of the precursor during which the boundary conditions are varied with time.

\section{III.B. Precursor Phase 2}

After the appropriate starting conditions were established through trial and error, we proceeded to simulate the actual measurement period from 8:15 to 11:26 a.m. The simulation from phase 1 was continued with a time-varying hub-height wind speed and direction dictated by radar data. These time-varying quantities determine the driving pressure gradient, which is updated at each time step to maintain a velocity magnitude and direction specified a priori. From examination of the output from the three different lidar-mounted temperature sensors (Fig. 4), we estimate a constant heating rate of $0.4^{\circ} \mathrm{K} / \mathrm{hr}$ over the simulated measurement period. Figure 7 summarizes all measurements discussed in Section II and the corresponding results from phase 2 .

In terms of wind speed and wind direction, the SOWFA results demonstrate excellent agreement with measured data (Fig. 7a \& b). The estimated shear exponent has the correct trend and magnitude up to 2 hours into the measurement period (Fig. 7c), after which correlations are less clear. At this time, it may be necessary to account for changes in the temperature inversion height. The effects of ground heating can be observed in the temperature and TI histories: the average temperature near the ground approaches the minimum values observed by the near-ground sensors, whereas higher off-the-ground temperature values approach the minima observed on the met mast (Fig. 7d). Higher near-ground temperatures correspond to higher average turbulence levels, which have increased slightly from their phase 1 values and are directly comparable to the corresponding measured values (Fig. 7e). At hub height, the extreme TI values are similar to measurements and the average differs by approximately $3 \%$. 

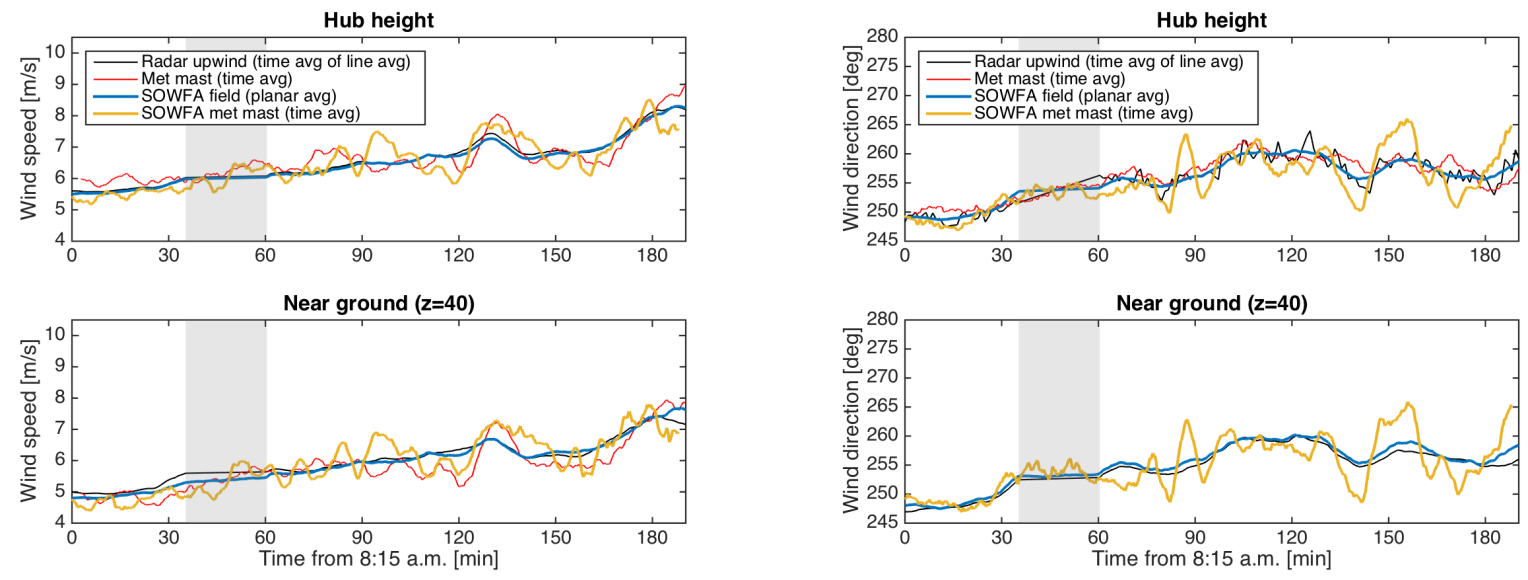

(a) Wind Speed

(b) Wind Direction

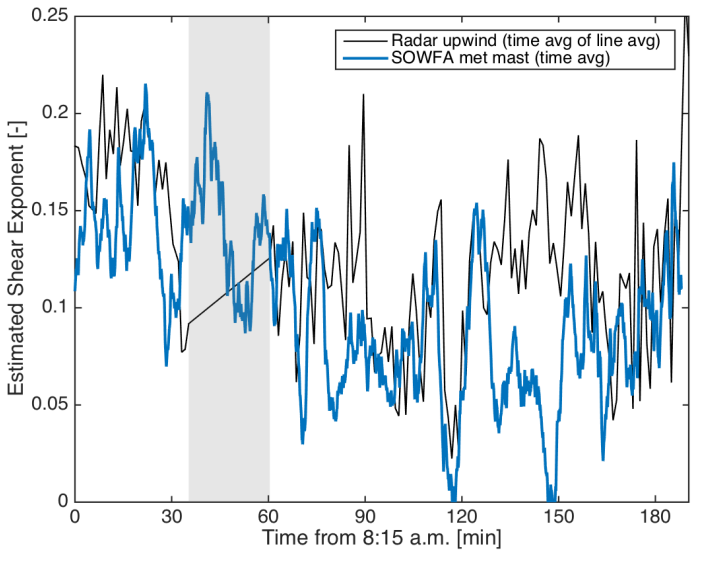

(c) Shear exponent

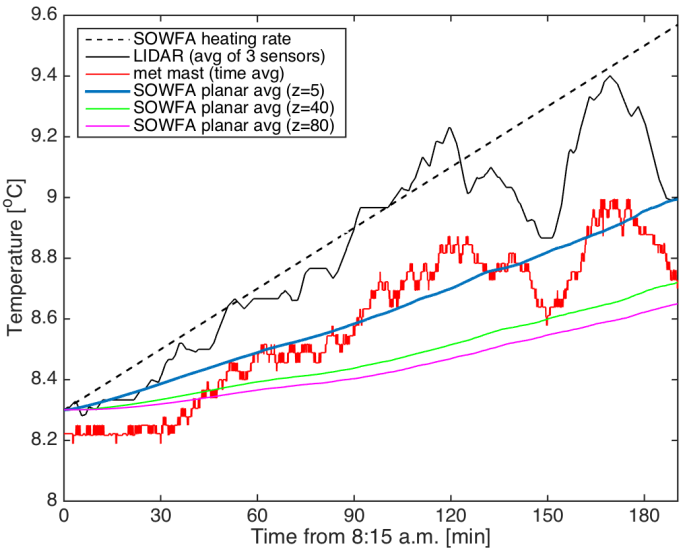

(d) Temperature
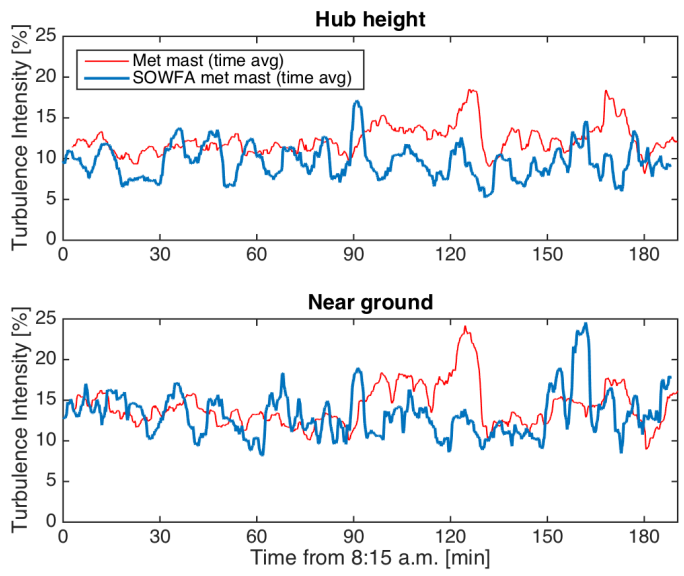

(e) Turbulence Intensity

Figure 7. Simulated results from precursor phase 2.

This report is available at no cost from the National Renewable Energy Laboratory (NREL) at www.nrel.gov/publications. 
Table 3. Averaged power statistics.

\begin{tabular}{lcc}
\hline & Avg. Normalized T89 Power & Avg. Normalized T90 Power \\
\hline SOWFA & 0.17 & 0.10 \\
SOWFA w/FAST & 0.16 & 0.10 \\
Measured & 0.16 & 0.09 \\
\hline
\end{tabular}

Table 4. Averaged rotor speed statistics.

\begin{tabular}{lcc}
\hline & Avg. Normalized T89 RPM & Avg. Normalized T90 RPM \\
\hline SOWFA & 0.63 & 0.56 \\
SOWFA w/FAST & 0.63 & 0.56 \\
Measured & 0.62 & 0.55 \\
\hline
\end{tabular}

\section{Turbine Simulations}

A specific subset of turbines within the wind plant was identified from the radar measurements for further investigation. This subset consisted of two turbines, labeled T89 and T90 in Fig. 1, which are positioned in the northwest corner of the measured radar domain. These two turbines were selected because turbine T90 is immediately downwind of turbine T89, which allows us to examine the power output and velocity profiles for a directly waked turbine. Both turbines T89 and T90 are also relatively close to the inlet plane, which allows them to be studied independently of the other turbines. These two turbines were simulated using SOWFA and the precursor inflow previously developed in Section III. The first 30 minutes of the turbine operations were simulated, starting from 8:15 a.m. local time, during which the wind direction and speed remained approximately constant, and the measured temperature showed few effects from heating. The simulated performance of the two turbines was then compared to the recorded data.

\section{IV.A. Computational Configuration}

Simulations of this turbine subset were performed using two different methods within SOWFA. The first method used SOWFA with a simple actuator line model representation of the turbine and a speed-torque table that governs the operation of the turbine. ${ }^{5}$ The second method couples the SOWFA model to FAST, ${ }^{13}$ which simultaneously computes the blade structural behavior and turbine system response to the aerodynamics supplied by the SOWFA computations. Furthermore, the second method also uses the same turbine controller as the measured turbines.

Both simulation types used the same physical domain as the precursor simulations ( $5 \mathrm{~km}$ by $3 \mathrm{~km}$ by $1 \mathrm{~km}$ ), but included a refined mesh surrounding the turbines to resolve the blade-level aerodynamics. In the far field, 10-m resolution was used, similar to the resolution of the precursor simulations. Closest to the turbine, the mesh resolution was increased to $1.25 \mathrm{~m}$, giving a total mesh size of approximately 40 million cells. The time-step size was also reduced to a constant 0.02 seconds, leading to a maximum Courant number of approximately 0.2 . The west and south inlet planes used data captured from the phase 2 precursor simulation as boundary conditions to generate the same atmospheric state.

Both turbines T89 and T90 were set at a constant fixed yaw position of 250 degrees relative to north. This closely matches the hub-height wind direction for the initial 30 minutes (Fig. 7). During the initialization phase of the main simulation, the turbines adjust to the local velocity field and start to develop velocity deficits behind the turbine rotors. Approximately 300 seconds after the start of the simulation, fully developed wakes are established. After this point, turbine and velocity field statistics are collected and analyzed against measured data.

\section{IV.B. Results}

The performance of turbine T89 and T90, as predicted by the SOWFA simulations, is shown in Figs. 8 and 9. The overall behavior of the turbine subset is captured relatively well. In comparison with the upstream turbine, the downstream T90 turbine spends a majority of this time period at near the minimum 
Turbine 89

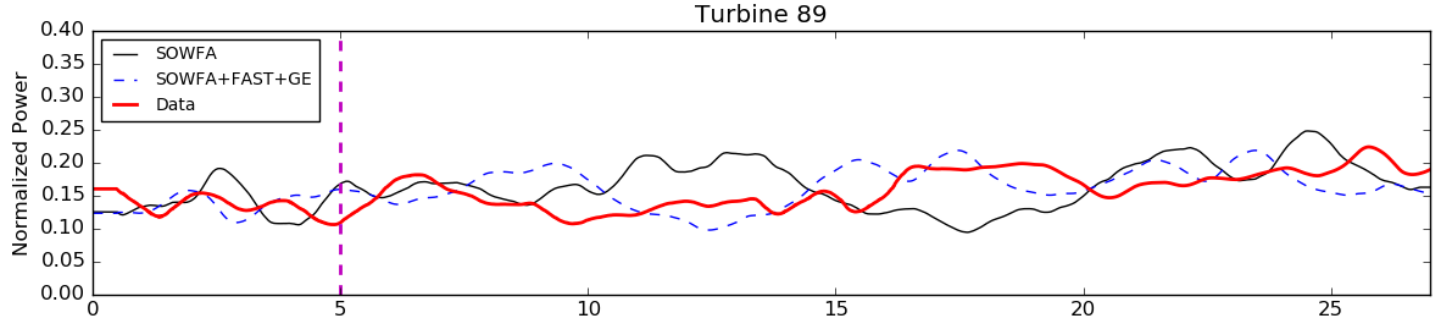

Turbine 90

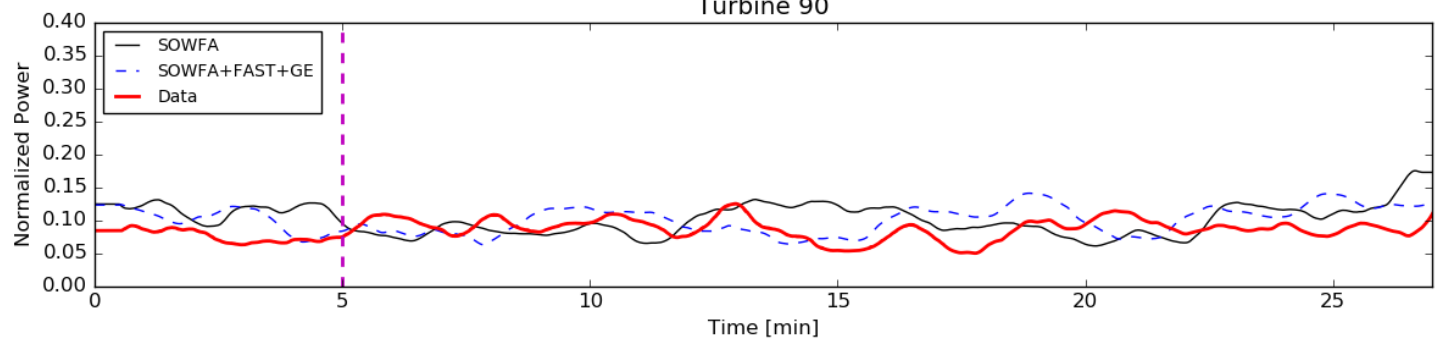

Figure 8. One-minute-averaged generator power from turbine T89 and T90.

Turbine 89

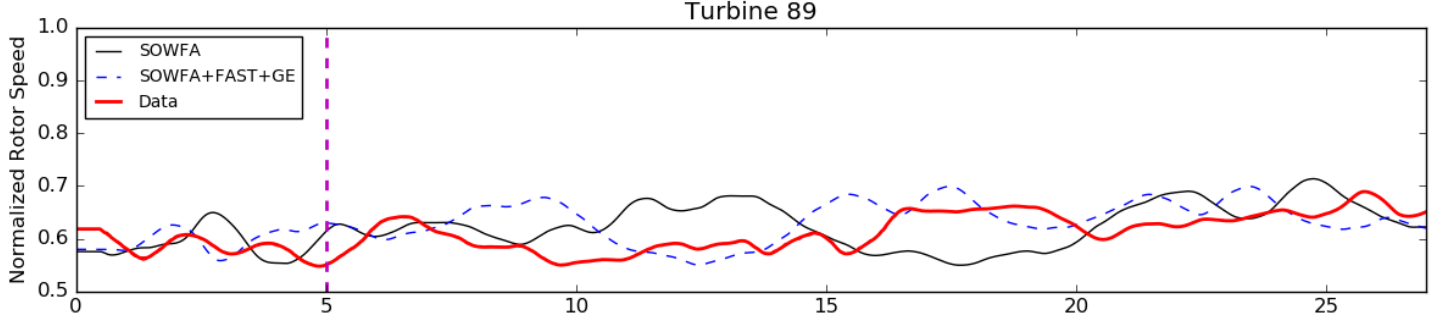

Turbine 90

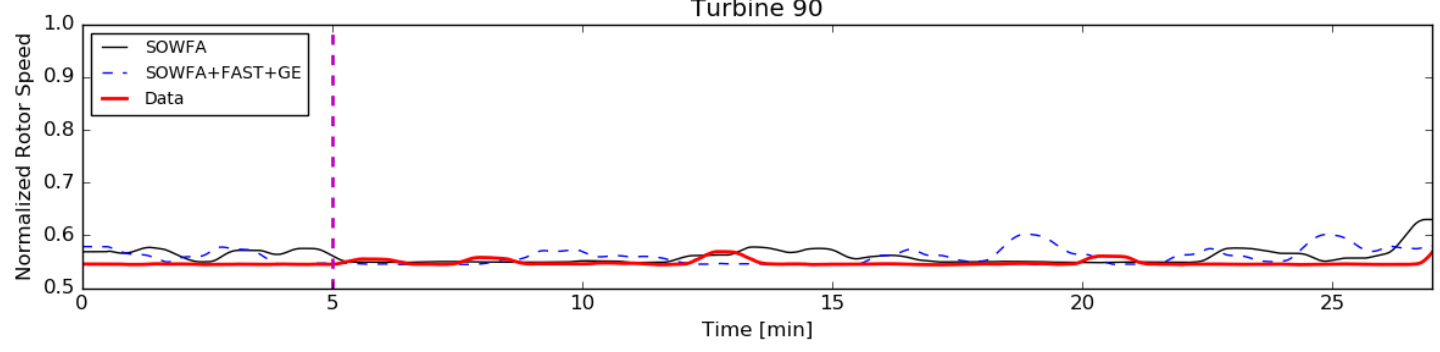

Figure 9. One-minute-averaged rotor speed from turbine T89 and T90. 

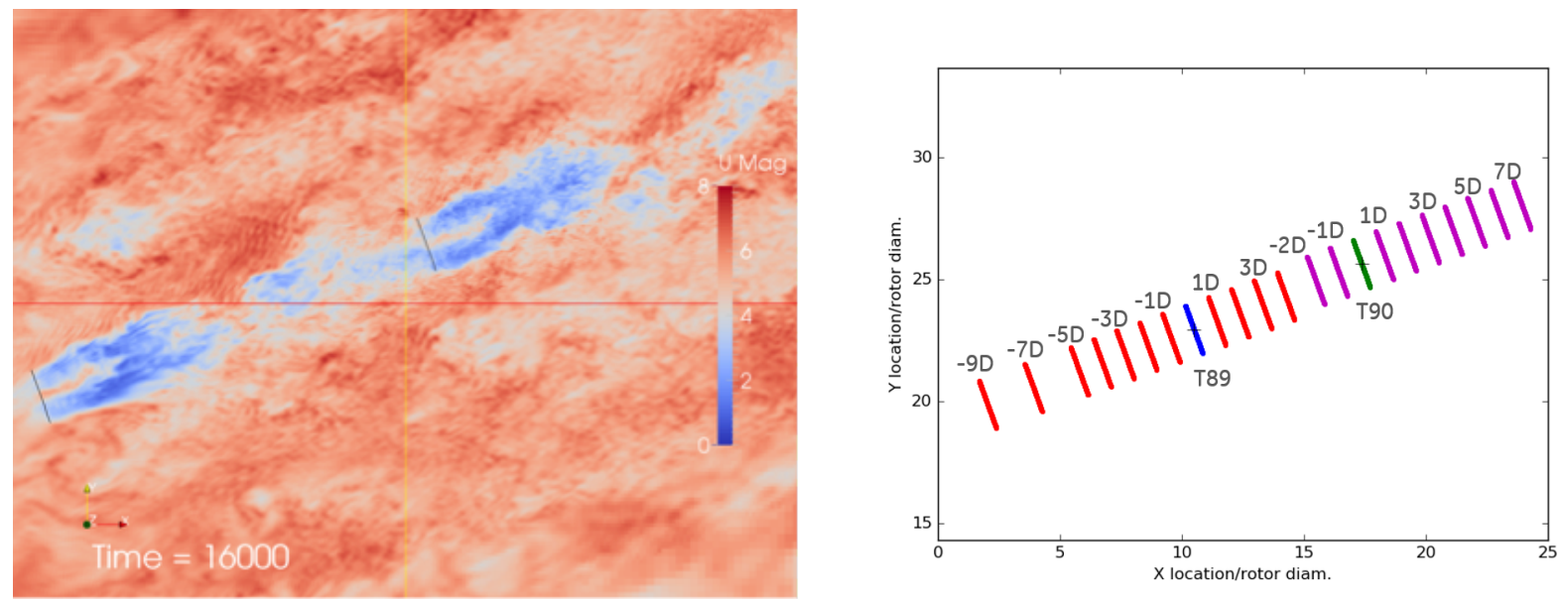

Figure 10. [left] Instantaneous snapshot of the velocity field at hub height near turbine T89 and T90. [right] Sampling locations upstream and downstream of both turbines.

cut-off speed, and consequently produces approximately $60 \%$ less power. This decrease is reflected in both the isolated SOWFA model and the FAST-coupled SOWFA simulations. For the upstream T89 turbine, the overall averaged power and rotor speed matched the measured data to within $3 \%$ for both SOWFA simulations (see Tables 3 and 4). Although rotor speeds were also predicted within the same margin for the downstream T90 turbine, the SOWFA simulations overpredicted the power output by approximately $10 \%$.

In addition to the turbine performance, the wake velocities surrounding the turbines can also be compared to the radar data. Hub-height velocity profiles were sampled at various locations upstream and downstream of both turbines, illustrated in Fig. 10. The results from both the radar measurements and the simulated velocity profiles upstream of T89 are relatively uniform. However, the radar velocities are approximately $0.5-$ $0.8 \mathrm{~m} / \mathrm{s}$ higher than SOWFA predictions at the particular inflow locations for these two turbines. It should be noted that the radar introduces significant smoothing in its data processing algorithms, and comparisons should be limited to a qualitative nature at this point.

Comparisons of the wakes downstream of both turbines are shown in Figs. 11 and 12. The nondimensional velocity deficits at the hub-height planes were computed using as:

$$
U_{\Delta}=\frac{U_{i n}-U}{\left\langle U_{i n}\right\rangle}
$$

where the inflow $\left\langle U_{i n}\right\rangle$ is the averaged horizontal velocity over the entire sampling location 1 rotor diameter upstream of each turbine. From these comparisons, we find little difference in the wake behavior between the noncoupled and FAST-coupled SOWFA simulations. These results also show the qualitative similarity between the radar-measured wakes and LES-computed wakes. Immediately downstream of both turbines at $2 D$, the simulated velocity deficit at the wake center differs from the radar average by $12 \%$. Farther downstream the wake decay between SOWFA and the radar measurements is similar, and the wake depth and width are approximately captured by the LES calculations. Emulating the radar data processing when evaluating the SOWFA flow field produces a more diffuse wake with greater wake width and reduced velocity deficit. However, it is unclear whether or not this accounts for the horizontal offset in the wake at $2 D$ and $3 D$, and quantifying the exact sampling-induced error is not possible given limitations in available data.

\section{Conclusions}

In this work, we demonstrated the ability to use LES to replicate both the atmospheric boundary layer and turbine performance of an operating wind farm in real-world conditions. This was done using the SOWFA tool to generate a precursor simulation for the turbulent wind farm inflow which was tuned to measured non-equilibrium wind conditions at the site during a specified time window. Overall, comparisons to measurements showed that SOWFA was able to reasonably capture the mean wind speed, wind direction, turbulence intensity, and temperature behavior of the atmospheric boundary layer. We believe that the multistage precursor approach demonstrated here may be readily applied to more complex wind farm 

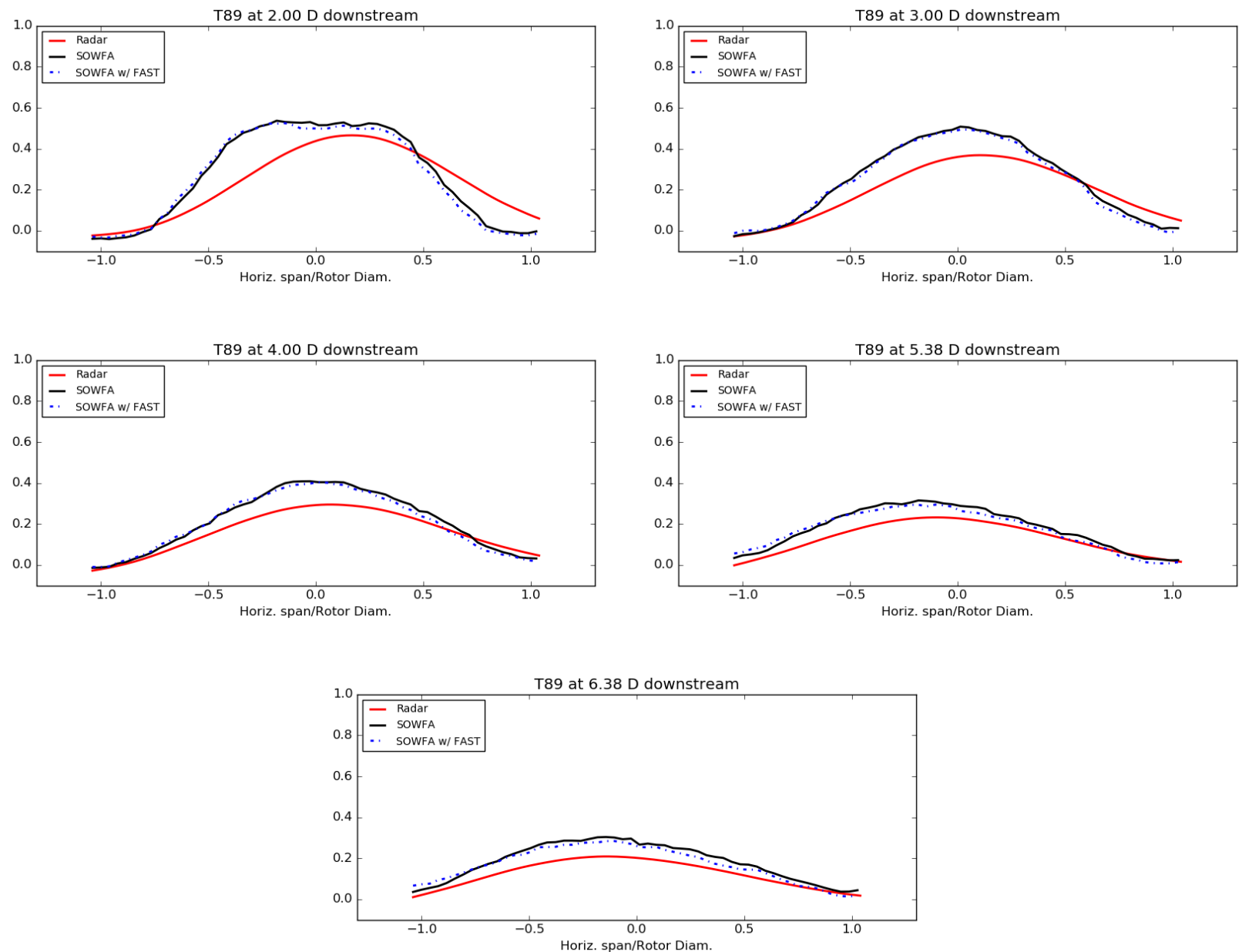

Figure 11. Wake velocity deficit $U_{\Delta}$ downstream of turbine 89 , measured at the hub-height planes $2,3,4,5.38$, and 6.38 diameters downstream of the rotor.
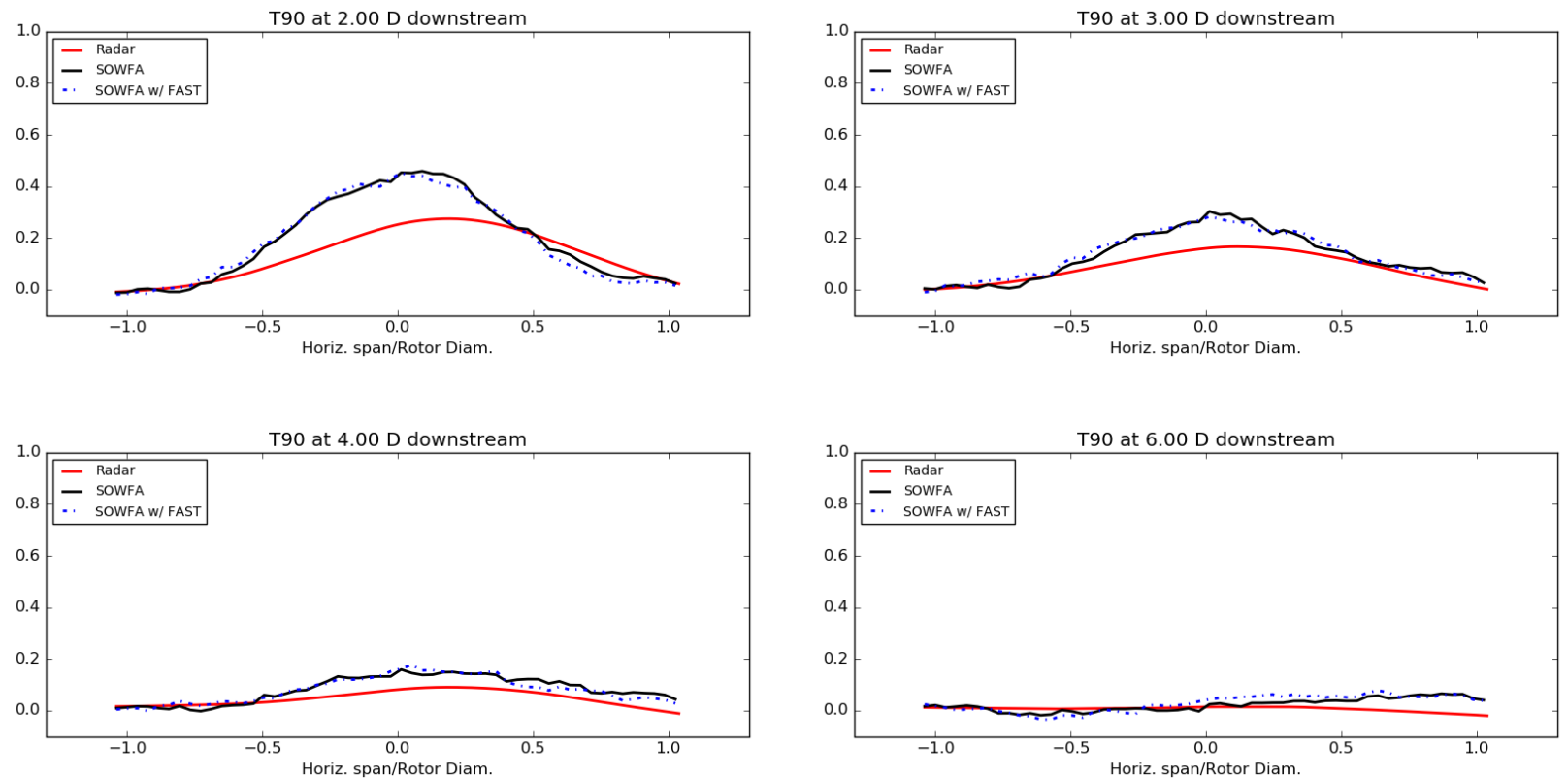

Figure 12. Wake velocity deficit $U_{\Delta}$ downstream of turbine 90, measured at the hub-height planes $2,3,4$, and 6 diameters downstream of the rotor. 
configurations with non-equilibrium atmospheric conditions.

A variety of data were considered for tuning the precursor simulation. The met mast provided better temporal resolution and permitted more accurate quantitative assessments of the turbulence intensity compared to other measurements. However, the spatially averaged radar data were more representative of the wind farm atmospheric conditions as a whole (e.g., with respect to the estimated shear). Because agreement with all measured quantities at every measurement height is not guaranteed, we focused our efforts on matching the turbulence level at hub height to the actual met mast data; farm-level atmospheric stability (approximately neutral) was inferred using shear profiles from the radar data. Surface roughness was not very important in this case, given uncertainties in the actual ground conditions of the site and the minimal effect this quantity had on the average turbulence level. Lastly, although the inversion height was kept constant in this study, it should be increased for more unstable conditions or if a complete diurnal cycle is to be considered.

The precursor simulation was then used to drive an aeroelastic simulation of the wind turbines using NREL's FAST tool coupled with SOWFA. This simulation focused on two turbines in a waked configuration during the initial time period. The simulated performance of these two turbines was compared to the measured turbine performance, and demonstrated that SOWFA was able to accurately capture the performance loss of the waked turbine. Ongoing work includes evaluation of additional subsets of turbines in the wind plant during other periods within the measurement campaign. We believe that the agreement between measured and simulated performance would not be possible given the apparent wake disparities, and expect that the measured wakes contain an appreciable amount of sampling-induced errors. In the future, we intend to directly model the radar sampling within our simulations to facilitate comparisons between measurement and simulation.

\section{Acknowledgments}

This work was supported by the U.S. Department of Energy under Contract No. DE-AC36-08GO28308 with the National Renewable Energy Laboratory. Funding for the work was provided by the DOE Office of Energy Efficiency and Renewable Energy, Wind and Water Power Technologies Office. Andreas Herrig from GE Global Research provided guidance on processing the field measurements. Sang Lee from NREL provided additional FAST modeling support.

The U.S. Government retains and the publisher, by accepting the article for publication, acknowledges that the U.S. Government retains a nonexclusive, paid-up, irrevocable, worldwide license to publish or reproduce the published form of this work, or allow others to do so, for U.S government purposes.

\section{References}

\footnotetext{
${ }^{1}$ Ivanell, S. S. A., Numerical Computations of Wind Turbine Wakes, Ph.D. thesis, KTH, Stockholm, 2009.

${ }^{2}$ Churchfield, M. J., Lee, S., Moriarty, P. J., Martinez, L. A., Leonardi, S., Vijayakumar, G., and Brasseur, J. G., "A Large-Eddy Simulation of Wind-Plant Aerodynamics," 50th AIAA Aerospace Sciences Meeting, Nashville, Tennessee, Jan. 2012.

${ }^{3}$ Stevens, R. J. A. M., Gayme, D. F., and Meneveau, C., "Effect of Turbine Alignment on the Average Power Output of Wind-Farms," Lyngny, 2013, pp. 611-623.

${ }^{4}$ Churchfield, M. J., Lee, S., Moriarty, P. J., Hao, Y., Lackner, M. A., Barthelmie, R., Lundquist, J. K., and Oxley, G., "A Comparison of the Dynamic Wake Meandering Model, Large-Eddy Simulation, and Field Data at the Egmond Aan Zee Offshore Wind Plant," 33rd Wind Energy Symposium, American Institute of Aeronautics and Astronautics, 2015.

${ }^{5}$ Churchfield, M. J., Lee, S., Michalakes, J., and Moriarty, P. J., "A Numerical Study of the Effects of Atmospheric and Wake Turbulence on Wind Turbine Dynamics," Journal of Turbulence, Vol. 13, Jan. 2012, pp. N14.

${ }^{6}$ Calaf, M., Meneveau, C., and Meyers, J., "Large Eddy Simulation Study of Fully Developed Wind-Turbine Array Boundary Layers," Physics of Fluids, Vol. 22, No. 015110, 2010.

${ }^{7}$ Churchfield, M. and Lee, S., "Simulator for Wind Farm Applications," available online at https://nwtc.nrel.gov/SOWFA, accessed May 19, 2016, created by the National Renewable Energy Laboratory.

${ }^{8}$ Jonkman, J. M. and Buhl, M. L., "FAST User's Guide," Tech. Rep. NREL/EL-500-38230, National Renewable Energy Laboratory, Aug. 2005.

${ }^{9}$ Wharton, S. and Lundquist, J. K., "Atmospheric Stability Affects Wind Turbine Power Collection," Environmental Research Letters, Vol. 7, No. 1, 2012, pp. 014005.

${ }^{10}$ Manwell, J. F., McGowan, J. G., and Rogers, A. L., Wind Energy Explained: Theory, Design and Application, 2nd Edition, John Wiley \& Sons Ltd., 2009.

${ }^{11}$ Vanderwende, B. and Lundquist, J. K., "Could Crop Height Affect the Wind Resource at Agriculturally Productive Wind Farm Sites?" Boundary-Layer Meteorology, Vol. 158, 2016, pp. 409-428.
} 
${ }^{12}$ Basu, S., Holtslag, A. A. M., Wiel, B. J. H. V. D., Moene, A. F., and Steeneveld, G.-J., "An Inconvenient "truth" about Using Sensible Heat Flux as a Surface Boundary Condition in Models under Stably Stratified Regimes," Acta Geophysica, Vol. 56, No. 1, March 2008, pp. 88-99.

${ }^{13}$ Lee, S., Churchfield, M., Moriarty, P., Jonkman, J., and Michalakes, J., "Atmospheric and Wake Turbulence Impacts on Wind Turbine Fatigue Loadings," 50th AIAA Aerospace Sciences Meeting, Nashville, Tennessee, January 9-12, 2012. 